\title{
Effect of Heat Treatments on Thermally Formed Martensite Phases in Monocrystalline Cu-Al-Ni Shape Memory Alloy
}

\author{
Hidekazu SAKAMOTO and Ken'ichi SHIMIZU
}

The Institute of Scientific and Industrial Research, Osaka University, Mihoga-oka, Ibaraki, Osaka-fu, 567 Japan.

(Received on September 12, 1988; accepted in the final form on November 18, 1988)

\begin{abstract}
The effect of heat treatments on martensitic transformations in monocrystalline Cu-14.1Al-4.2Ni (mass\%) (alloy 1) and Cu-14.7Al-4.2Ni (mass\%) (alloy 2) shape memory alloys has been investigated by means of differential scanning calorimetry measurements, optical microscopy and tensile tests of martensitic specimens. As a result, it was found in alloy 1 that not only transformation temperatures shifted but also thermally formed martensite changed from $\beta_{1}{ }^{\prime}$ to $\gamma_{1}{ }^{\prime}$ with heat treatment. Meanwhile, in alloy 2 , the $\gamma_{1}^{\prime}$ martensite was thermally $f^{\prime}$ ormed regardless of heat treatment. To clarify the reason for the change of thermally formed martensite, equilibrium teinperatures, To, for the $\beta_{1} \rightleftarrows \gamma_{1}{ }^{\prime}$ and $\beta_{1} \rightleftarrows \beta_{1}{ }^{\prime}$ transformations and their variation with heat treatment were estimated by utilizing previous data on stress-induced martensitic transformations and associated pseudoelasticity of the alloys (Trans. Jpn. Inst. Met., 28 (1987), 264). Consequently, by taking into consideration the variation of To temperatures and, in addition, supercooling neccessary for the nucleation of the $\gamma_{1}^{\prime}$ and $\beta_{1}{ }^{\prime}$ martensites, the origin was successfully elucidated in terms of a simple scheme that the martensite phase with the highest $M_{S}$ temperature changed from the $\beta_{1}{ }^{\prime}$ to the $\gamma_{1}{ }^{\prime}$ with heat treatment. Applicability of this scheme was discussed on similar phenomena occurred in other alloy systems in which martensite phases changed with chemical composition.
\end{abstract}

KEY WORDS: martensitic transformation; copper-aluminum-nickel alloy; heat treatment; temperature hysteresis; differential scanning calorimetry; shape memory alloy; equilibrium temperature; pseudoelasticity; phase stability; transformation temperature.

\section{Introduction}

It has been known in many alloy systems that crystal structure of martensite phase varies with the alloy composition. For example, in $\mathrm{Cu}-\mathrm{Al}$ alloys the martensite phase changes from $\beta^{\prime}$ (disordered fcc) to $\gamma_{1}^{\prime}$ $(2 \mathrm{H})$ via $\beta_{1}^{\prime}(18 \mathrm{R})$ with increasing $\mathrm{Al}$ content, ${ }^{1-3)}$ in $\mathrm{Au}-\mathrm{Cd}$ alloys from $\beta_{2}^{\prime}(9 \mathrm{R})$ to $\zeta_{2}^{\prime}$ (trigonal) via $\gamma_{2}^{\prime}(2 \mathrm{H})$ with increasing $\mathrm{Cd}$ content, ${ }^{4-8}$ ) and in $\mathrm{Fe}-\mathrm{Mn}$ alloys from $\alpha$ (fcc) to $\varepsilon$ (hcp) with increasing Mn content., ${ }^{9,10}$ These phenomena have been taken for granted, although the fundamental origins for the changes are not known.

On the other hand, it has been found in a $\mathrm{Au}-$ $47.5 \mathrm{at} \% \mathrm{Cd}$ alloy more than thirty years ago that crystal structure of martensite phase changes with heat treatment. ${ }^{11}$ That is, $\beta_{2}$ (B2) parent phase transforms into the $\gamma_{2}^{\prime}$ martensite when the alloy is slowly cooled from a high temperature. However, the transformation from $\beta_{2}$ to the $\zeta_{2}^{\prime}$ martensite takes place, either when initially quenched to a temperature above $M_{s}$ and subsequently cooled to a temperature below $M_{f}$, or when quenched to a temperature below $M_{f}$ followed by heating untill the as-quenched martensite is reversely transformed and subsequently cooled to a temperature below $M_{f}$. Many investigations have so far been performed on this phenomenon, ${ }^{12-14)}$ but the origin for the phase change with heat treatment has not been clarified yet.
Following the observation in the Au-Cd alloy, Hull and Garwood ${ }^{15}$ found a similar phenomenon in a $\mathrm{Cu}-12.8 \mathrm{Al}-7.7 \mathrm{Ni}(\operatorname{mass} \%)$ alloy such that when the quenched alloy is cooled, the $\beta_{1}^{\prime}$ martensite is formed, but that when the quenched alloy is tempered and subsequently cooled, the $\gamma_{1}^{\prime}$ martensite is formed. They explained the cause of the phenomenon as follows: On tempering, segregation occurred by forming $\mathrm{Al}$ rich and poor regions, which accompanied by the formation and growth of ordered domains. Then, the $\gamma_{1}^{\prime}$ martensites formed in the $\mathrm{Al}$ rich region propagated into the $\mathrm{Al}$ poor region. However, their explanation is inconsistent with the fact that $M_{s}$ temperature decreases with increasing $\mathrm{Al}$ content. Similar phenomenon was observed in a $\mathrm{Cu}-$ $11.8 \mathrm{Al}-4.0 \mathrm{Mn}(\mathrm{mass} \%)$ alloy by Sugino et al., and they gave a similar explanation. ${ }^{16)}$ Then, the reason for the change of thermally formed martensite phases with heat treatment may not be said to be clarified.

Recently, the present authors investigated the effect of heat treatments on stress-induced martensitic transformations (SIMT) and associated pseudoelasticity (PE) in monocrystalline $\mathrm{Cu}-\mathrm{Al}-\mathrm{Ni}$ alloys. ${ }^{17)}$ In the course of the investigation, similar change of martensite phases with heat treatment has been observed. That is, when a $\mathrm{Cu}-14.1 \mathrm{Al}-4.2 \mathrm{Ni}(\mathrm{mass} \%)$ alloy was rapidly quenched from the high temperature $\beta$ phase region, transformation into the $\beta_{1}^{\prime}$ martensite takes place on cooling, whereas, when the alloy was normal- 
ly quenched or when it was thereafter aged at $473 \mathrm{~K}$, transformation into the $\gamma_{1}^{\prime}$ martensite occurs on cooling. However, no detailed examination has been made on the effect of heat treatments on characteristics of thermally formed martensites. The present study has been conducted to provide more positive evidence for the change of thermally formed martensites with heat treatment. The martensitic transformations in the specimens subjected to different heat treatments have been examined by means of differential scanning calorimetry (DSC) measurements, optical microscopy and tensile tests of martensitic specimens. Results of the examination and the reason for the change of martensite phases will be described in the present paper.

\section{Experimental Procedure}

Experimental procedures, such as alloy preparation method, heat treatment and tensile test, were almost the same as those in the previous investigation. ${ }^{17}$ Hence, they will be described only briefly in the following, along with the procedures for DSC measurements and optical microscopy newly utilized in the present study.

The alloys used were alloy $1(\mathrm{Cu}-14.1 \mathrm{Al}-4.2 \mathrm{Ni}$ $(\operatorname{mass} \%))$ and alloy $2(\mathrm{Cu}-14.7 \mathrm{Al}-4.2 \mathrm{Ni}(\operatorname{mass} \%))$. Single crystals, whose orientations were within a few degrees from $\langle 001\rangle$, were grown by a modified Bridgman method. Plate specimens suitable for tensile tests were spark cut from the single crystals and were subjected to the following three kinds of heat treatments;

(a) $1273 \mathrm{~K} \rightarrow$ Quenched into $10 \% \mathrm{NaOH}$ solution,

(b) $1273 \mathrm{~K} \rightarrow$ Quenched into water, and

(c) aged at $523 \mathrm{~K}$ for $3.6 \mathrm{ks}$ after treatment (b). The above three kinds of heat treatments will be abbreviated here as R.Q., N.Q. and A., respectively.

Some of the plate specimens subjected to the above heat treatments were cut into slices from which 3 $\mathrm{mm} \phi$ discs for DSC measurements were spark cut and electropolished. DSC measurements were made at a scanning rate of $10 \mathrm{~K} / \mathrm{s}$. Martensite microstructure was optically observed on specimens of alloy 1 for which DSC measurements and tensile tests were made. R.Q. and N.Q. specimens, whose $M_{s}$ temperatures were around or below room temperature, were subzero cooled in ethylene glychol monoethyl ethel and ethyl alcohol, respectively, and their martensite microstructures were observed. Tensile tests were performed only on the sub-zero cooled R.Q. specimens of alloy 1 , by using Instron machine.

\section{Experimental Results}

\subsection{DSC Curves}

Fig. 1 shows DSC curves of alloy 1 subjected to the three kinds of heat treatments. The measurements were started from a cooling run followed by a heating run, and they were repeated in this sequence. The numbers in parentheses at the left hand side of

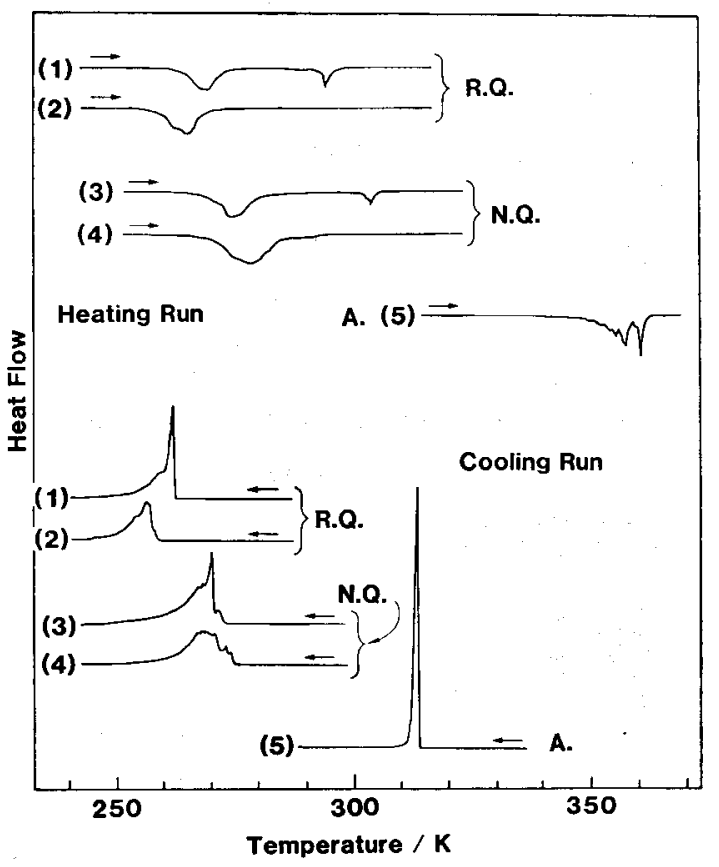

Fig. 1. Effect of heat treatments on DSC curves of alloy 1, (1), (2): R.Q., (3), (4): N.Q. and (5): A.

each curve are inserted to show the correspondence between the cooling and heating curves. Two curves are shown for R.Q. and N.Q. specimens, since different behavior was observed from specimen to specimen. It is seen from the figure that not only peak temperatures but also peak shape change with the heat treatments. The peak temperatures rise in the order of R.Q., N.Q. and A. specimens. This is in accordance with the results in the previous study, ${ }^{17)}$ in which transformation temperatures were obtained by the observation of surface relief effect.

The peak shapes may be classified into the following three different types depending on the heat treatments. The first type is seen on curves (1) and (3), in which a low sharp peak accompanied by tail at the lower temperature side appear on cooling, but two separate peaks on heating. The second type is seen on curves (2) and (4), in which a broad peak appears both on cooling and heating runs. These two types of DSC curves have been observed both in R.Q. and N.Q. specimens. The third type is seen on curve (5), in which a high sharp peak appears both on cooling and heating runs, although the peak height on heating run are somewhat lower than that on cooling. The difference in peak shape is due to the formation of different martensite phases, as will be explained below.

Fig. 2 shows the variation of DSC curves with the heat treatments for alloy 2. The peak temperatures rise in the order of the R.Q., N.Q. and A. specimens, as in alloy 1. However, regardless of the heat treatments, high sharp peak appears both on cooling and heating runs, being different from alloy 1 .

\subsection{Microstructural Observation}

In order to identify the transformation products responsible for the different peak features in DSC 
curves, martensite microstructure of alloy 1 was observed by optical microscopy. However, as mentioned in the previous section, R.Q. and N.Q. specimens showed different DSC curves from specimen to specimen. The microstructure must then be observed by using just the specimen whose DSC curve have been measured. Hence, another specimen of R.Q. and N.Q. ones was supplied for DSC measurements, whose curves are shown in Fig. 3. In the cooling curve of R.Q. specimen, a low broad peak

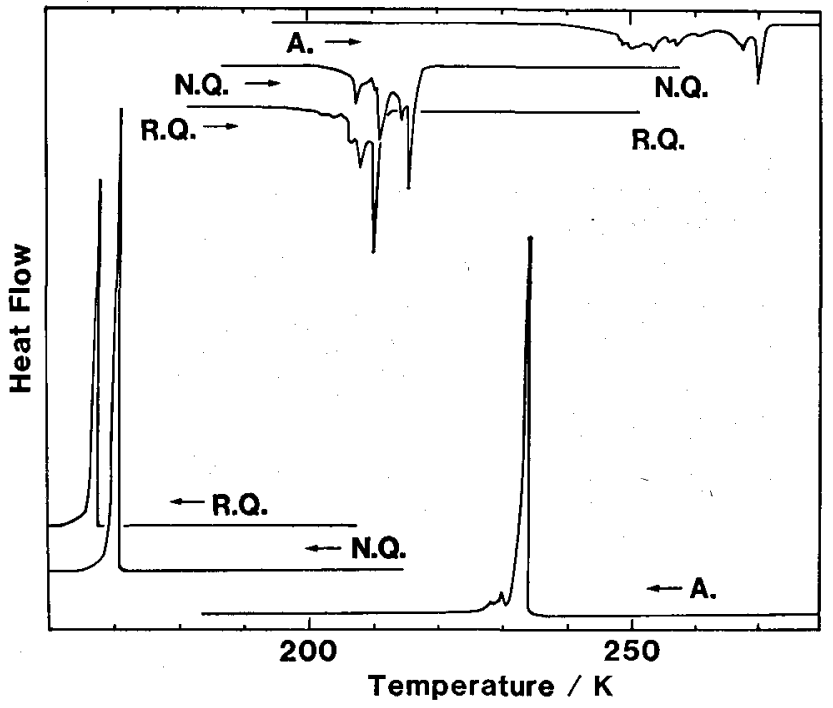

Fig. 2. Effect of heat treatments on DSG curves of alloy 2. appears just like curve (2) in Fig. 1. On the other hand, the DSG peak observed in the N.Q. specimen is different from curve (3) in Fig. 1. That is, a high sharp peak is observed, although a small sub-peak appears on the higher temperature side. Moreover, the main peak does not accompany by no obvious tail on the lower temperature side. Thus, the peak feature is rather similar to that for A. specimens.

By using such specimens of alloy 1, the martensite microstructures of the quenched states were observed at around $235 \mathrm{~K}$ under three kinds of magnifications, and they are shown in Fig. 4 along with those of $\mathrm{A}$. specimen observed at room temperature. It is obviously seen from the figure that the martensite microstructures also change with heat treatment. In R.Q. specimen, (a), $\left(a^{\prime}\right)$ and $\left(a^{\prime \prime}\right)$, are observed self-ac-

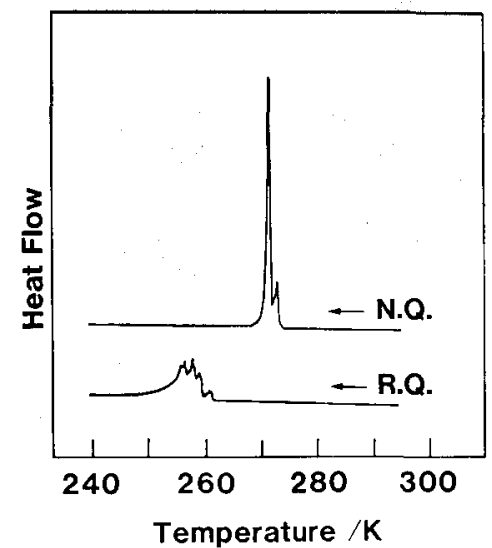

Fig. 3.

DSC curves of R.Q. and N.Q. specimens of alloy 1 , whose martensite microstructure thereafter optically observed are shown in Fig. 4.

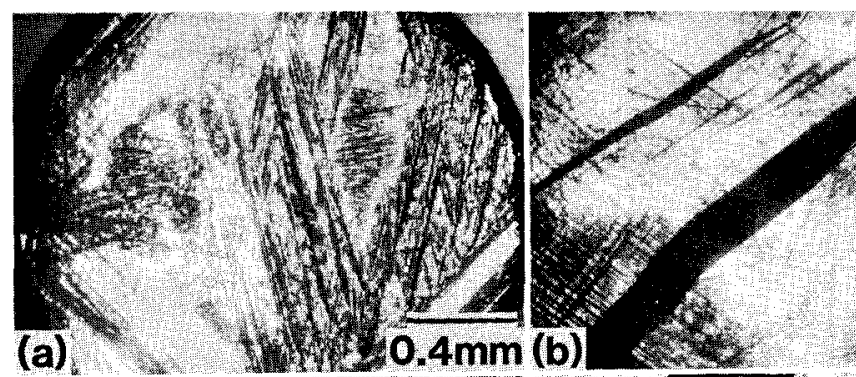

(a)



(a")

(a), (a'), (a') : R.Q.

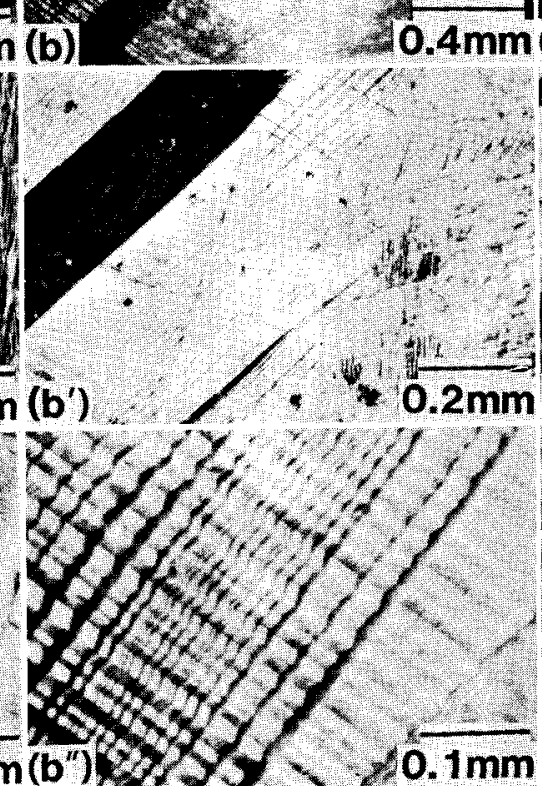

(b), $\left(b^{\prime}\right),\left(b^{\prime \prime}\right):$ N.Q.

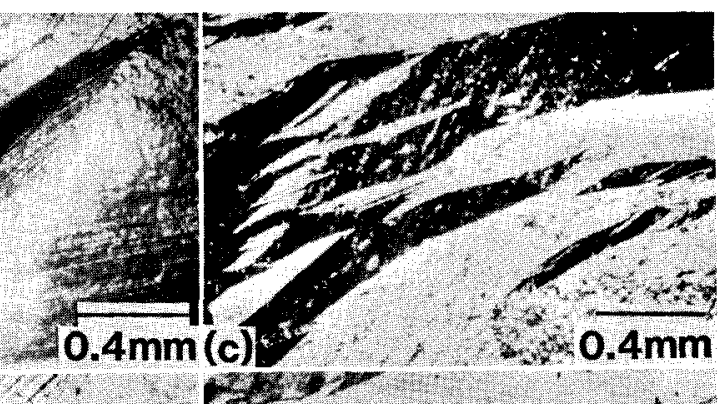

serte

(x)

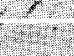


comodating zig-zag groups of four martensite variants, which are typical of the $\beta_{t}^{\prime}$ martensite. ${ }^{18,19}$ ) Hence, the second type low broad peak in DSC curves may be ascribed to the formation of the $\beta_{1}^{\prime}$ martensite. On the other hand, both in N.Q., (b), ( $\left.b^{\prime}\right)$ and $\left(b^{\prime \prime}\right)$, and A. specimens, (c), ( $\left.\mathrm{c}^{\prime}\right)$ and $\left(\mathrm{c}^{\prime \prime}\right)$, are observed coarse martensite plates with internal twins, which is typical of the $\gamma_{1}^{\prime}$ martensite. ${ }^{20,21)}$ Thus, the third type high sharp peak in DSC curves may evidently be attributed to the formation of the $\gamma_{1}^{\prime}$ martensite. Similarly, the thermally formed martensites in alloy 2 are the $\gamma_{1}^{\prime}$ martensites regardless of the heat treatments. The transformations responsible for the first type DSG curves will be discussed in Sec. 3.4.

The $\beta_{1}^{\prime}$ and $\gamma_{1}^{\prime}$ martensites were observed also in tensile specimens of the R.Q. and N.Q. alloy 1 , respectively. The progress of the $\beta_{1} \rightarrow \beta_{1}^{\prime}$ and $\beta_{1} \rightarrow \gamma_{1}^{\prime}$ transformations in R.Q. and N.Q. tensile specimens has been observed by cooling down (and heating up) in an ethyl alcohol bath, the microstructural change being shown in Figs. 5 and 6 . In the R.Q. specimen
(Fig. 5) whose transformation temperatures for the $\beta_{1}^{\prime}$ martensite* were $M_{s}=333 \mathrm{~K}, M_{f}=268 \mathrm{~K}$ and $A_{f}=$ $345 \mathrm{~K}$, the transformation has proceeded in a partitioning way. That is, a set of thin long parallel plates of the $\beta_{1}^{\prime}$ martensite appears when the specimen was immersed into ethyl alcohol bath as in (a). On further cooling, another set of thin parallel plates is formed crossing the preexisting set of the plates, (b). Thereafter, many fine plates are formed in the regions surrounded by the two sets of plates, (c) to (e).

On the other hand, in the N.Q. specimen (Fig. 6) whose transformation temperatures are $M_{s}=266 \mathrm{~K}$, $A_{s}=275 \mathrm{~K}$ and $A_{f}=323 \mathrm{~K}, \gamma_{1}^{\prime}$ martensite with typical spear-like morphology is observed, as has been observed by many investigators. ${ }^{15,22-24)}$ The $\gamma_{1}^{\prime}$ martensite plate seen on the left hand side of (a) easily grows with slight decrease of temperature, and no other plate has nucleated in this field of view, (b) to (d). The reverse movement of the interface easily occur as well with slight increase of temperature, (e) to (h). It is thus suggested from the comparison of Fig. 5

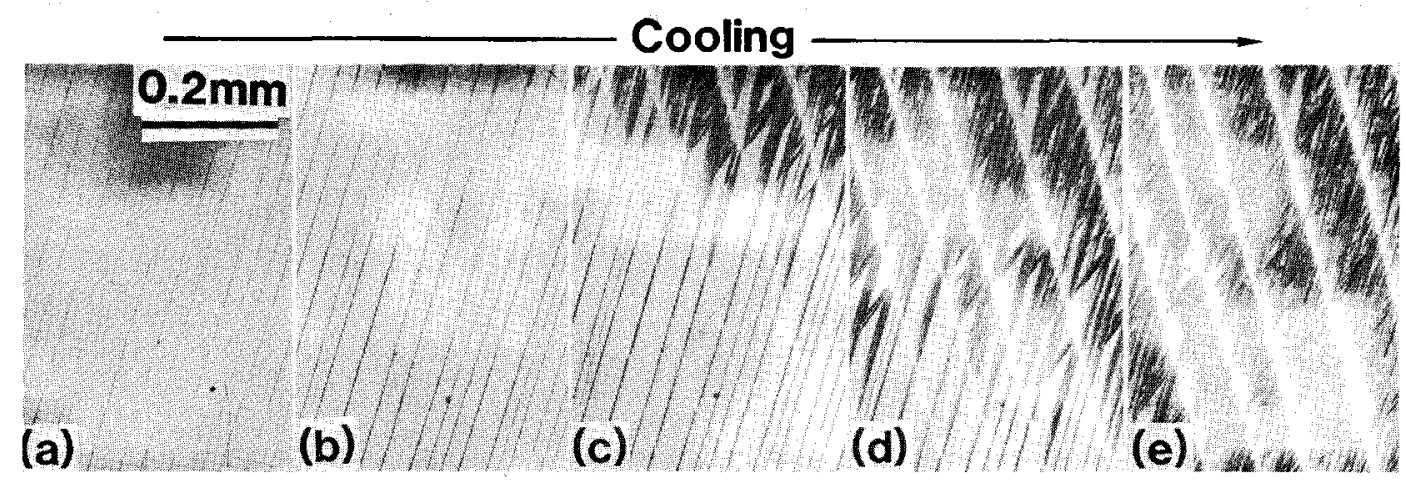

Fig. 5. Microstructural change during cooling an R.Q. tensile specimen of alloy 1.

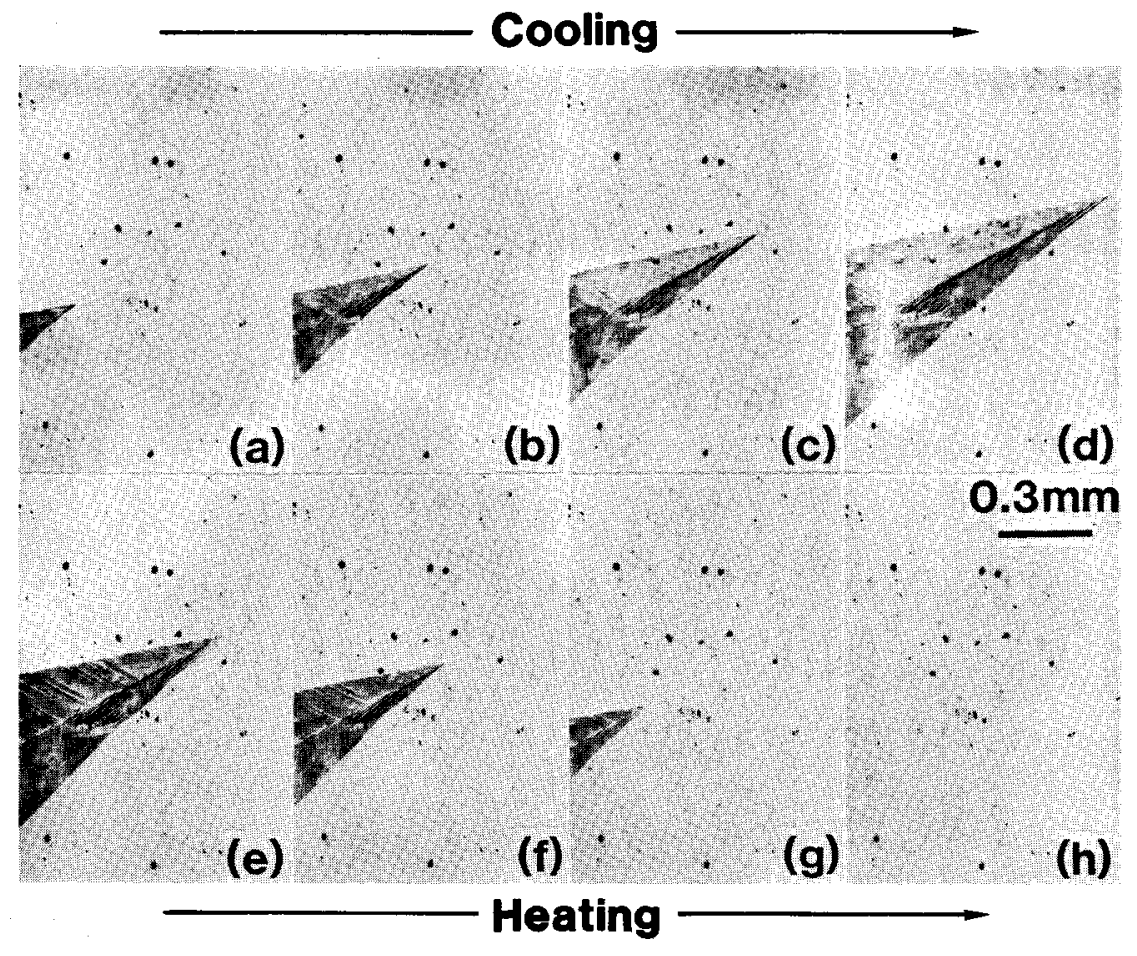

Fig. 6.

Microstructural change during cooling, (a) to (d), and heating, (d) to (h), an N.Q. specimen of alloy 1 .

* In this specimen, the $\gamma_{1}^{\prime}$ martensite was also observed, as verified in Sec. 3.3. 
with Fig. 6 that the $\beta_{1}^{\prime}$ and $\gamma_{1}^{\prime}$ martensites are quite different in the nucleation and growth behavior. That is, the $\beta_{1}^{\prime}$ martensite casily nucleates but the growth is difficult, whereas the $\gamma_{1}^{\prime}$ martensite does not easily nucleate but the growth is easy after once nucleated. This behavior is in consistent with the previous observation on stress-induced $\beta_{1} \rightarrow \beta_{1}^{\prime}$ and $\beta_{1} \rightarrow \gamma_{1}^{\prime}$ transformations. ${ }^{25}$ )

\subsection{Tensile Deformation of Martensitic Specimens}

In the previous section, the kind of thermally formed martensites has been identified only through optical microscopy observation. However, we have obtained another evidence for the formation of the $\beta_{1}^{\prime}$ martensites in R.Q. specimens by means of tensile tests of martensitic specimens. Fig. 7 shows a stressstrain curve for an R.Q. specimen of alloy 1 at $253 \mathrm{~K}$ $\left(<M_{f}\right)$. The curve shows clearly two stages on loading, although a little bend appears at $\sim 250 \mathrm{MPa}$ in the linear portion between the two stages. The first non-pseudoelastic stage is, of course, due to reorientation of multivariant martensites, and the elongation on the first stage reaches $\sim 6.5 \%$. On the other hand, the second pseudoelastic stage, which is due to some stress-induced martensite-to-martensite transformation, appears at $\sim 500 \mathrm{MPa}$, and the amount of strain yielded on the second stage reaches $\sim 13 \%$.

Such stress-strain behavior is well rationalized as follows by supposing that thermally formed martensite is the $\beta_{1}^{\prime}$. The first stage strain, $\sim 6.5 \%$, is attainable through reorientation of the thermally formed multivariant $\beta_{1}^{\prime}$ martensites, but not of the $\gamma_{1}^{\prime}$ martensites, because the strain yielded through reorientation of multivariant martensites should be the same order as that associated with stress-induced martensitic transformation above $M_{s}$. For the present specimens with nearly $\langle 100\rangle$ orientation, calculated transformation elongations associated with the $\beta_{1} \rightarrow \beta_{1}^{\prime}$ and $\beta_{1} \rightarrow \gamma_{1}^{\prime}$ transformations are 8.4 and $4.3 \%$, respectively. ${ }^{25)}$ In fact, only $\sim 3.5 \%$ strain has previously been obtained (see Fig. 2(B) of Ref. 26)) for the reorientation of $\gamma_{1}^{\prime}$ martensites in a specimen subjected to almost the same treatment as the present N.Q. alloy 1 . Second, the stress for the appearance of the second stage, 497
$\mathrm{MPa}$, is appropriate for the occurrence of the $\beta_{1}^{\prime} \rightarrow \alpha_{1}^{\prime}$ transformation, because critical stresses for the $\gamma_{1}^{\prime} \rightarrow \beta_{1}^{\prime \prime}$ and $\beta_{1}^{\prime \prime} \rightarrow \alpha_{1}^{\prime}$ transformations at $253 \mathrm{~K}$ have previously been found ${ }^{17)}$ to be around 275 and $500 \mathrm{MPa}$, respectively. Moreover, the amount of strain yielded on the second stage, $\sim 13 \%$, is in accordance with experimentally measured elongation for the $\beta_{1}^{\prime}\left(\beta_{1}^{\prime \prime}\right) \rightarrow \alpha_{1}^{\prime}$ transformation, $11.5 \sim 14.5 \%$, ${ }^{7)}$ but not achievable through the $\gamma_{1}^{\prime} \rightarrow \beta_{1}^{\prime \prime}$ transformation $\left(\sim 3.5 \%{ }^{17}\right)$ ).

However, a few part of thermally formed martensites may be the $\gamma_{1}^{\prime}$ martensite, because the little bend observed at $\sim 250 \mathrm{MPa}$ in the linear portion between the two stages is thought to be due to the $\gamma_{1}^{\prime} \rightarrow \beta_{1}^{\prime \prime}$ transformation. Thus, we can conclude that the most of thermally formed martensites in R.Q. specimen is the $\beta_{1}^{\prime}$ martensite, although a few part may be the $\gamma_{1}^{\prime}$ martensite.

\subsection{Transformations Responsible for the First Type Peak}

We now discuss on the transformations responsible for the first type low sharp peak accompanied by a tail on cooling run and separated two peaks on heating run in DSC curves of R.Q. and N.Q. specimens. Since the peak feature on cooling run seems to be an intermediate between the second type broad peak and the third type high sharp peak, it is surmised that the $\beta_{1}^{\prime}$ and $\gamma_{1}^{\prime}$ martensites are simultaneously formed on cooling. Consequently, two separate peaks on heating run may be ascribed to the fact that the $\beta_{1}^{\prime} \rightarrow \beta_{1}$ and $\gamma_{1}^{\prime} \rightarrow \beta_{1}$ reverse transformations have separately taken place, possibly due to a different temperature hysteresis between the $\beta_{1} \rightleftarrows \beta_{1}^{\prime}$ and $\beta_{1} \rightleftarrows \gamma_{1}^{\prime}$ transformations. Temperature hysteresis of these two transformations may be known from curves (2), (4) and (5) in Fig. 1. However, in order to illustrate it more clearly, martensite amount vs. temperature $(f-T)$ diagrams are deduced from the DSC curves, (2) and (5), which are typically ascribed to the $\beta_{1} \rightleftarrows \beta_{1}^{\prime}$ and $\beta_{1} \rightleftarrows \gamma_{1}^{\prime}$ transformations, respectively, because partial change of enthalpy is thought to be proportional to the transformed fraction. In Figs. 8 are shown the ob:ained diagrams, from which the temperature hysteresis for the $\beta_{1} \rightleftarrows \gamma_{1}^{\prime}$ transformations, $\sim 50 \mathrm{~K}$, is seen to be obviously larger than that for the $\beta_{1} \rightleftarrows \beta_{1}^{\prime}$ transformations,

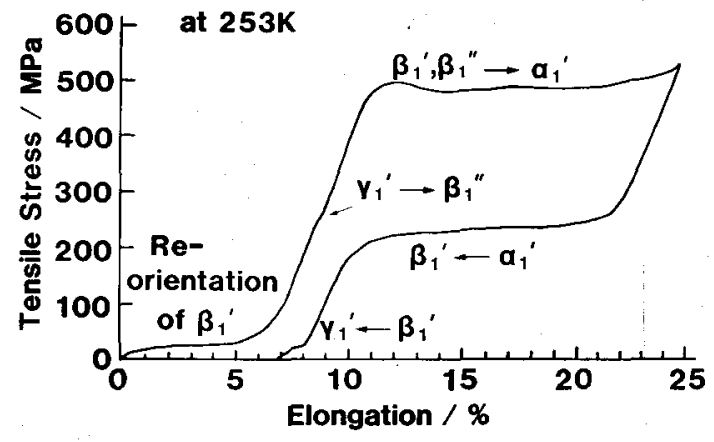

Fig. 7. Stress-strain curves of an R.Q. specimen of alloy 1 at $253 \mathrm{~K}$.

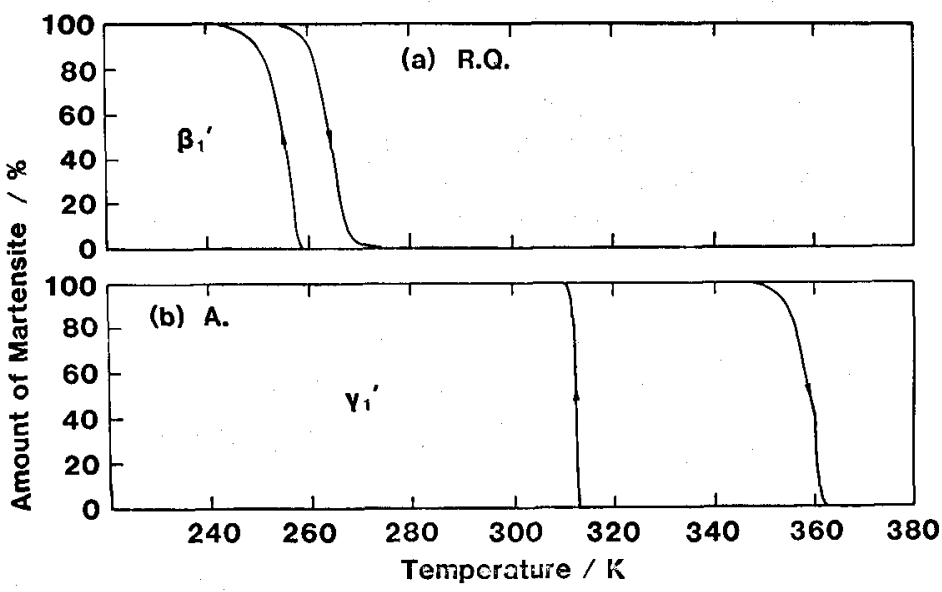

Fig. 8. Transformed fraction vs. temperature $(f-T)$ diagram of alloy 1, (a) R.Q. specimen, (b) A. specimen. 
$\sim 15 \mathrm{~K}$. Similar phenomenon has been observed in $\mathrm{Cu}-\mathrm{Zn}-\mathrm{Al}$ alloys as well. ${ }^{27)}$ Thus, the low temperature broad peaks and high temperature sharp peaks on heating run in the curves (1) and (3) in Fig. I may be attributed to the $\beta_{1}^{\prime} \rightarrow \beta_{1}$ and $\gamma_{1}^{\prime} \rightarrow \beta_{1}$ reverse transformations, respectively. Such separated two peaks on heating run have recently been observed in a poly-crystalline $\mathrm{Cu}-13.99 \mathrm{Al}-3.5 \mathrm{Ni}(\operatorname{mass} \%)$ alloy by Humbeeck et al. ${ }^{28)}$

Next, we will briefly compare characteristics of the $\beta_{1} \rightleftarrows \beta_{1}^{\prime}$ transformations with those the $\beta_{1} \rightleftarrows \gamma_{1}^{\prime}$ transformations, on the basis of the $f-T$ diagrams. The $f-T$ diagrams may be featured by two factors, temperature hysteresis and overall transformation temperature range, $\delta T\left(M_{s}-M_{f}\right.$ on cooling and $A_{f}-A_{s}$ on heating). The temperature hysteresis has commonly been explained to be originated from the nucleation processes of a martensite phase in a parent phase and of the parent phase in the martensite phase. ${ }^{29-31)}$ Thereby, the driving force necessary for the nucleation of the $\gamma_{1}^{\prime}$ martensite is expected to be larger than that of the $\beta_{1}^{\prime}$ martensite. On the other hand, $\delta \mathcal{T}$ is thought to be originated from a nonchemical elastic resistive force to the movement of martensite interfaces, according to a viewpoint of the balance between chemical and elastic forces. ${ }^{32)} \quad$ Since $\delta T$ for the $\beta_{1} \rightleftarrows$ $\beta_{1}^{\prime}$ is larger than that for the $\beta_{1} \rightleftarrows \gamma_{1}^{\prime}$, the growth of the $\gamma_{1}^{\prime}$ is expected to be easier than that of the $\beta_{1}^{\prime}$. These are in agreement with the inference made from the microstructural observation.

\section{Discussion}

\subsection{Basic Concept for the Change of Martensite Phases}

Before going into discussion, the basic concept of our idea for the change of martensite phases will be shown in the following. It has been reported in various alloy systems that transformation temperatures shift with heat treatment. For example, it was found in a $\mathrm{Fe}_{3} \mathrm{Pt}$ alloy system that transformation temperatures of the alloy quenched from $\sim 1500 \mathrm{~K}$ decreased with increasing annealing time at $\sim 900 \mathrm{~K}$, and that the annealing brought about an increase of degree of order. ${ }^{34)}$ The reason was qualitatively explained in such a way that the chemical free energy $G$ of the austenite was more decreased than that of the martensite $^{35)}$ with the progress of ordering. In $\mathrm{Cu}-\mathrm{Al}-\mathrm{Ni}$ alloys, on the contrary, slow quenching ${ }^{36)}$ or aging at low temperature ${ }^{17)}$ increased transformation temperatures, and was found to bring about an increase of degree of order. Thus, the effect of degree of order on transformation temperatures is opposite in the $\mathrm{Fe}_{3} \mathrm{Pt}$ and $\mathrm{Cu}-\mathrm{Al}-\mathrm{Ni}$ alloys. We speculate the reason such that the progress of the ordering causes the stabilization of the close packed structures in both the alloys, i.e., a fcc structure of the austenite in $\mathrm{Fe}_{3} \mathrm{Pt}$ alloys while a $2 \mathrm{H}$ structure of the $r_{1}^{\prime}$ martensite in $\mathrm{Cu}-\mathrm{Al}-\mathrm{Ni}$ alloys.

Hence, the reason for the change of transformation temperatures in $\mathrm{Cu}-\mathrm{Al}-\mathrm{Ni}$ alloys will be considered to be analogous with that in the $\mathrm{Fe}_{3} \mathrm{Pt}$ alloy. Fig. 9 is a schematic $G$ vs. $T$ diagram, showing how heat treatments influence the diagram. The solid lines in the figure show the variation of $G$ of the parent, $G_{P}$, and of the martensite, $G_{M}$, with temperature, when the $\mathrm{Cu}-\mathrm{Al}-\mathrm{Ni}$ alloy is subjected to a heat treatment (ht. a), such as R.Q. which brings about an insufficient order (the martensite in this case is the $\beta_{1}^{\prime}$ ). Meanwhile, when the alloy is subjected to another heat treatment (ht. b) such as low temperature aging which causes an increase in degrec of order, $G_{M}$ is more decreased than $G_{P}$, as expressed by the dash-dotted lines. In other words, the variation of $G_{M}$ with changing heat treatment from ht. a to ht. $b, \delta G_{M}$ (ht. $a \rightarrow b)$, is larger than that of $G_{P}, \delta G_{P}(h t . a \rightarrow b)$. As a result, the equilibrium temperature may be increased from $T_{o}(h t . a)$ to $T_{o}(h t . b)$.

Here, we assume that there is another "latent" martensite phase (to be specific, the $\gamma_{1}^{\prime}$ ) with slightly higher $G$ than the $\beta_{1}^{\prime}$ martensite in the alloy subjected to $h t$. a. This situation is schematically shown in Fig. 10. The latent $r_{1}^{\prime}$ martensite is not thermally formed, since $\mathcal{T}_{o}$ (ht. $a: \beta_{1} \rightleftarrows \gamma_{1}^{\prime}$ ) is lower than $\mathcal{T}_{0}$ (ht. $a: \beta_{1} \rightleftarrows \beta_{1}^{\prime}$ ). However, if $G_{\gamma_{1}^{\prime}}$ is more decreased than $G_{\beta_{1}^{\prime}}$ with changing heat treatment from $h t$. $a$ to ht. $b$, i.e., if $\delta G_{\gamma_{1}^{\prime}}(h t . a \rightarrow b)$ is larger than $\delta G_{\beta_{1}^{\prime}}(h t . a \rightarrow b)$, then $T_{0}\left(h t . b: \beta_{1} \rightarrow \gamma_{1}^{\prime}\right)$ may become greatly higher than $T_{o}\left(h t . b: \beta_{1} \rightarrow \beta_{1}^{\prime}\right)$. In this situation, the $\gamma_{1}^{\prime}$ martensite may be thermally formed. Thus, the change of martensite phase with heat treatment is well explained.

\subsection{Estimation of $T_{0}$ Temperature}

In order to verify the above hypothesis, the variation of $\tau_{o}$ temperatures for the $\beta_{1} \rightleftarrows \gamma_{1}^{\prime}$ and $\beta_{1} \rightleftarrows \beta_{1}^{\prime}$ transformations with heat treatment will be quantitatively estimated here. $T_{0}$ temperatures for these transformations will be denoted by $T_{o}\left(\gamma_{1}^{\prime}\right)$ and $T_{o}$ $\left(\beta_{1}^{\prime}\right)$, respectively, hereafter. $T_{0}$ temperatures for thermoelastic martensitic transformations can be determined in a usual way, such as $T_{o}=1 / 2\left(M_{s}+A_{f}\right) .^{37)}$ However, $T_{o}$ temperature for the "latent" transformation, which does not occur on cooling, can not be estimated by such a usual way. Then, as another way to experimentally estimate $T_{o}$ temperature, data of SIMT and associated PE will be utilized. The effect of heat treatments on SIMT and associated PE has previously been investigated ${ }^{17)}$ by using the same monocrystalline alloys 1 and 2 with $\sim\langle 00 \mathrm{l}\rangle$ orientation.

Fig. 11 (solid lines) shows equilibrium stresses $\sigma^{E}$ vs. $\mathcal{T}$ relations $\left(\sigma^{E}\right.$ : the midpoint between critical stresses on loading and unloading) for various transformations in alloy 1 subjected to the three kinds of heat treatments, and Fig. 12 (solid lines) those in alloy 2. $T_{o}\left(\beta_{1}^{\prime}\right)$ can easily be determined by extrapolating the straight solid lines of $\sigma_{2}^{E *} v s . T$ relations to zero stress, since the extrapolated temperatures may be regarded to correspond to $T_{o}$ temperatures. On the other hand, $\sigma_{1}^{E *}$ for the $\beta_{1} \rightleftarrows \gamma_{1}^{\prime}$ transformations

* The subscripts 1,2 and 3 of $\sigma^{E}$ denotes the $\beta_{1} \rightleftarrows \gamma_{1}^{\prime}, \beta_{1} \rightleftarrows \beta_{1}^{\prime}$ and $\gamma_{1}^{\prime} \rightleftarrows \beta_{1}^{\prime}\left(\beta_{1}^{\prime \prime}\right)$ transformations. 


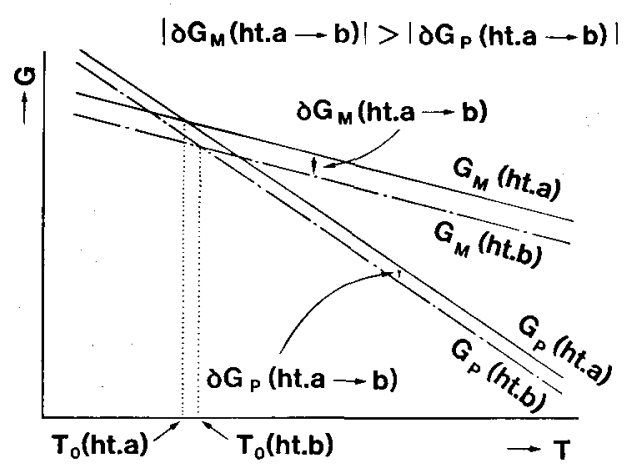

Fig. 9.

Schematic diagram showing the effect of heat treatments on chemical free energy of parent and martensite phases as a function of temperature.

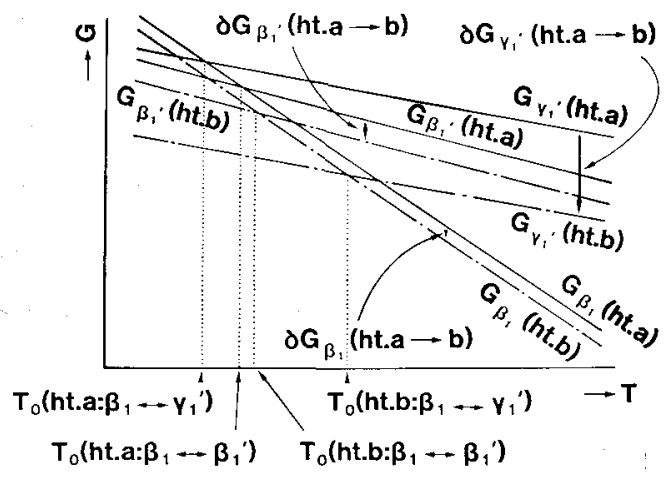

Fig. 10 .

Schematic diagram showing the effect of heat treatments on chemical free energy of $\beta_{1}$ parent, $\gamma_{1}^{\prime}$ and $\beta_{1}^{\prime}$ martensites as a function of temperature.

(termed transf. 1) are not shown in the figure, because the $\gamma_{1}^{\prime}$ martensite has not been stress-induced in alloy 1 or the stress-induced $\gamma_{1}^{\prime}$ has not reverse transformed in alloy 2 on unloading. Then, $T_{o}\left(\gamma_{1}^{\prime}\right)$ has been obtained by calculating $d \sigma_{1}^{E} / d T$ from the following equation, ${ }^{38)}$ which was deduced in a similar manner to the previous study ${ }^{39)}$ :

$$
\frac{d \sigma_{1}^{E}}{d T}=\left(\frac{d \sigma_{2}^{E}}{d T} \cdot \varepsilon_{2}-\frac{d \sigma_{3}^{E}}{d T} \cdot \varepsilon_{3}\right) / \varepsilon_{1},
$$

where, $T:$ temperature

$\varepsilon:$ transformation elongation.

Here, we assume that $\varepsilon$ is independent of the alloy compositions and the heat treatments, and that it is $8.4,4.3$ and $4.1 \%$ for transf. 1,2 and $3,{ }^{25}$ respectively. Table 1 shows the $d \sigma_{1}^{E} / d T$ calculated by substituting those $\varepsilon$ and the experimental $d \sigma_{2}^{E} / d T$ and

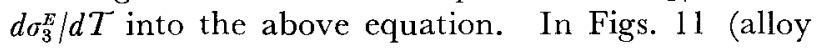
1) and 12 (alloy 2), the $\sigma_{1}^{E} v s$. Trelations (dash-dotted lines) thus obtained and the extrapolated $\sigma_{2}^{F}$ vs. $\mathcal{T}$ relation (dashed lines) are shown along with the measured $\sigma^{E}$ vs. $T$ relations (solid lines).

$\tau_{o}\left(\gamma_{1}^{\prime}\right)$ and $T_{o}\left(\beta_{1}^{\prime}\right)$ determined from these figures

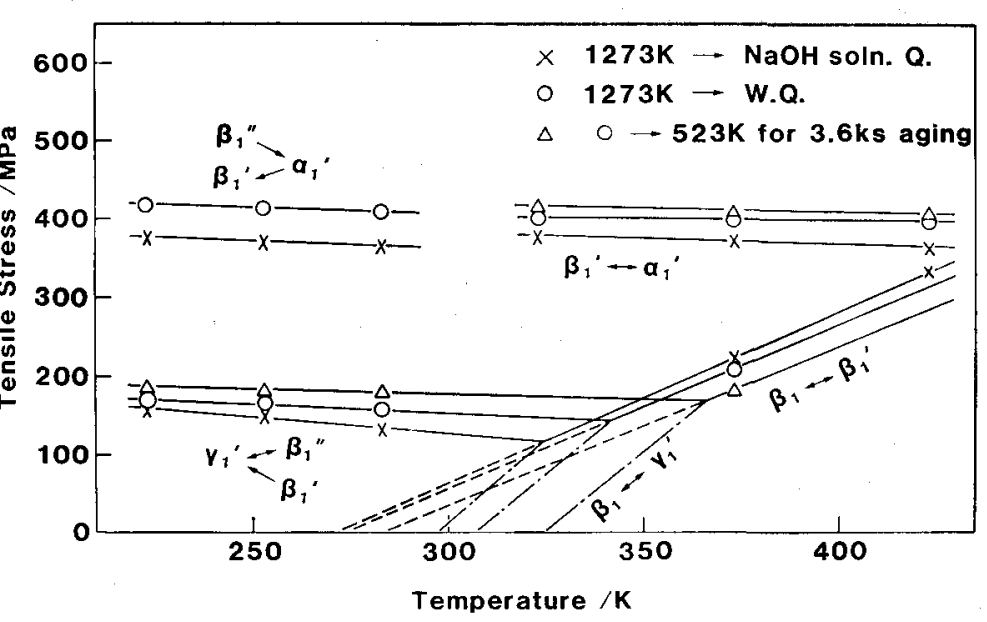

Fig. 11. Effect of heat treatments on equilibrium stress vs. temperature relation (including calculated (dash-dotted lines) and extrapolated (dashed lines) ones) for each transformation of alloy 1.

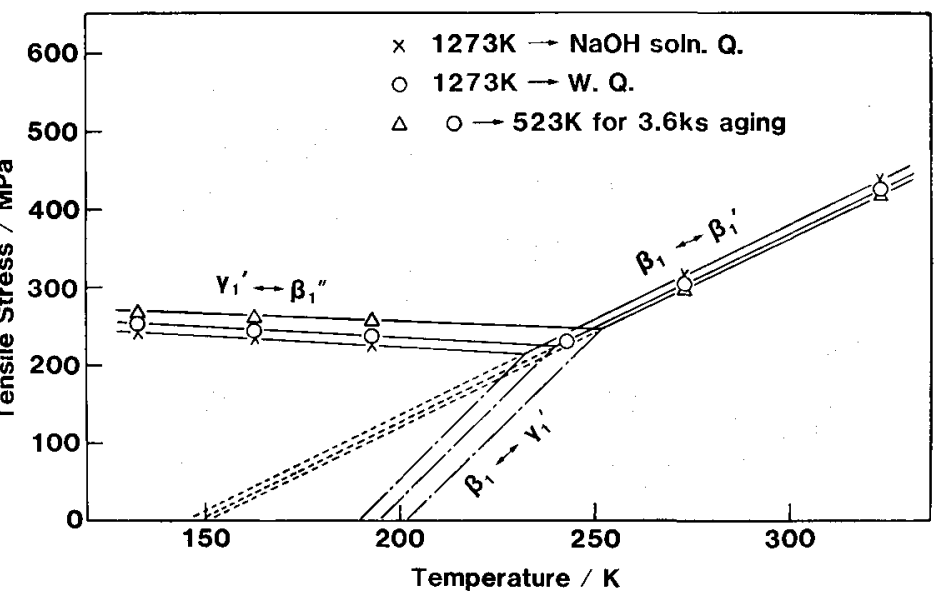

ig. 12. Effect of heat treatments on equilibrium stress vs. temperature relation (including calculated (dash-dotted lines) and extrapolated (dashed lines) ones) for each transformation of alloy 2.

Table 1. Observed and calculated temperature dependences of equilibrium stresses for the $\beta_{1}$ $\rightleftarrows \gamma_{1}^{\prime}, \beta_{1} \rightleftarrows \beta_{1}^{\prime}$ and $\gamma_{1}^{\prime} \rightleftarrows \beta_{1}^{\prime \prime}\left(\beta_{1}^{\prime}\right)$ transformations. $(\mathrm{MPa} / \mathrm{K})$

\begin{tabular}{llccc}
\hline & & $d \sigma_{2}^{E} / d T$ & $d \sigma_{3}^{E} / d T$ & $d \sigma_{1}^{E} / d T$ \\
(observed) & (observed) & (calculated) \\
\hline \multirow{3}{*}{ Alloy 1 } & R. Q. & 2.19 & -0.13 & 4.40 \\
& N.Q. & 2.09 & -0.05 & 4.13 \\
& A. & 2.03 & -0.10 & 4.06 \\
\hline \multirow{4}{*}{ Alloy 2 } & R. Q. & 2.45 & -0.29 & 5.06 \\
& N.Q. & 2.42 & -0.27 & 4.98 \\
& A. & 2.42 & -0.27 & 4.92 \\
\hline
\end{tabular}

are shown in Fig. 13, and the variation of $T_{o}$ temperatures with the heat treatments is clearly seen. The figure manifests that $T_{o}\left(\gamma_{1}^{\prime}\right)$ varies more markedly than $\mathcal{T}_{0}\left(\beta_{1}^{\prime}\right)$ with the heat treatments, although the variation of $T_{0}$ in alloy 2 is small. Consequently, the difference between $T_{o}\left(\gamma_{i}^{\prime}\right)$ and $T_{o}\left(\beta_{1}^{\prime}\right)$ appears to decrease in the order of A., N.Q. and R.Q. specimens, and $T_{o}\left(\gamma_{1}^{\prime}\right)$ are always higher than $T_{o}\left(\beta_{1}^{\prime}\right)$. However, by taking into account some supercooling necessary for the nucleation of two martensites, we 
can elucidate the reason that the thermally formed martensites in alloy 1 changes with heat treatment. Details will be discussed in Sec. 4.4.

It is to be noted here that $T_{o}$ temperatures estimated by the above method do not strictly coincide with those estimated by the usual method. This discrepancy may possibly be ascribed to some difference between thermally-induced and stress-induced transformations. However, it may be sure that the variation of $\mathcal{T}_{o}$ temperatures estimated by the above method with heat treatment qualitatively represent that of $T_{o}$ temperatures estimated by the usual method.

\subsection{Evaluation of $\Delta G_{P \rightarrow M}^{*}(0)$}

Now, the chemical free energy change associated with a transformation, $\Delta G^{*}$, and its variation with the heat treatments, $\delta \Delta G^{*}$, will further be evaluated. $G^{*}$ of a phase at a given temperature is approximated as a function of stress $\sigma$ (or force) by the following equation:

$$
G^{*}(\sigma)=G^{*}(0)-l^{\circ} \sigma,
$$

where, $G^{*}(0)$ : the chemical free energy,

$l^{\circ}:$ the length of the phase,

both in the absence of stress. ${ }^{39,40)}$

The following relation holds between the parent phase $P$ and the martensite phase $M$ at $\sigma^{E}$;

$$
G_{P}^{*}\left(\sigma^{E}\right)=G_{M}^{*}\left(\sigma^{E}\right)
$$

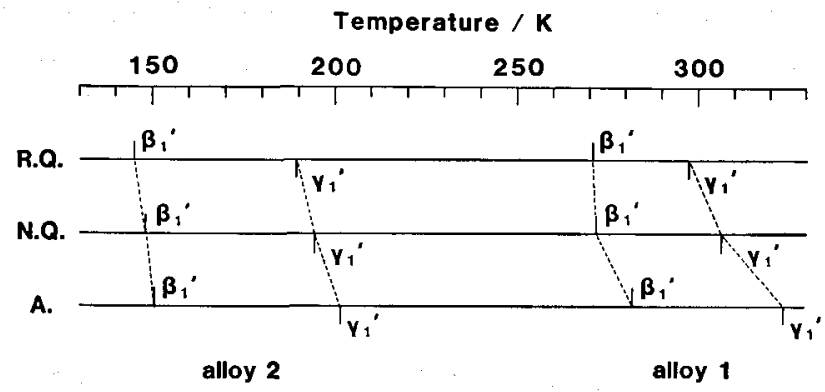

Fig. 13. Variation of $T_{o}$ temperatures with heat treatments, estimated by utilizing SIMT and associated PE.
Since the free energy change associated with the parent to martensite transformation under zero external stress, $\Delta G_{P \rightarrow M}^{*}(0)$, are expressed by

$$
\Delta G_{P \rightarrow M}^{*}(0)=G_{M}^{*}(0)-G_{P}^{*}(0),
$$

$\Delta G_{P \rightarrow M}^{*}(0)$ can be approximated by the following equation:

$$
\Delta G_{P \rightarrow M}^{*}(0) \simeq \Delta l_{P \rightarrow M}^{\circ} \cdot \sigma^{E},
$$

where, $\Delta l_{P \rightarrow M}^{\circ}:$ the elongation associated with the transformation (i.e., $\varepsilon$ ).

$\Delta G_{P \rightarrow M}^{*}(0)$ has been calculated for the $\beta_{1} \rightarrow \gamma_{1}^{\prime}$ and $\beta_{1}$ $\rightarrow \gamma_{1}^{\prime}$ transformations in alloys 1 and 2 , by using $\sigma^{E}$ shown in Figs. 11 and 12 and calculated $\Delta l^{\circ}$ (or $\varepsilon$ ). The results are shown in Fig. 14. The solid, dashdotted and dash-dot-dotted lines express $\Delta G_{P \rightarrow M}^{*}(0)$ curves for R.Q., N.Q. and A. specimens, respectively. The $\Delta G_{P \rightarrow M}^{*}(0)$ increases linearly with increasing temperature in accordance with the linear increase in $\sigma^{t}$. The increasing rate of $\Delta G_{P \rightarrow M}^{*}(0)$ against temperature is almost the same without regard of the transformations, alloys and heat treatments, but it is slightly larger for transf. 1 than for transf. 2 and for alloy 2 than for alloy 1.

Then, the variation of $\Delta G_{P \rightarrow M}^{*}(0)$ due to the change of heat treatment from I to II, $\delta \Delta G_{P \rightarrow M}^{*}(0 ; \mathrm{I} \rightarrow \mathrm{II})$, may be approximated by the equation

$$
\delta \Delta G_{P \rightarrow M}^{*}(0 ; \mathrm{I} \rightarrow \mathrm{II}) \simeq \Delta l_{P \rightarrow M}^{\circ}\left\{\sigma^{E}(\mathrm{II})-\sigma^{E}(\mathrm{I})\right\},
$$

where, $\sigma^{E}$ (I), $\sigma^{E}$ (II): the specimens subjected to the heat treatments $I$ and II, respectively.

Fig. 14 manifests that $\delta \Delta G_{\beta_{1} \rightarrow r_{1}^{\prime}}^{*}(0)$ is larger than $\delta \Delta G_{\beta_{1} \rightarrow \beta_{1}^{\prime}}^{*}(0)$ in both the alloys, and correspondingly that $T_{o}$ temperature, where $\Delta G_{P \rightarrow M}^{*}(0)=0$, for transf. 1 varies more than for transf. 2 with heat treatment. Table 2 shows numerical values of $\Delta G_{P \rightarrow M}^{*}$ ( 0 ; R.Q. $\rightarrow$ A.) for alloy 1 at $340 \mathrm{~K}$ and for alloy 2 at $240 \mathrm{~K}$. $\delta \Delta G_{\beta_{1} \rightarrow r_{1}^{\prime}}^{*}(0)$ is seen to be about twice of $\delta \Delta G_{\beta_{1} \rightarrow \beta_{1}^{\prime}}^{*}(0)$ in both the alloys, although those in alloy 2 are about half of those in alloy 1. From this calculation, it may be deduced that some structural alteration caused by changing heat treatment has, in general,

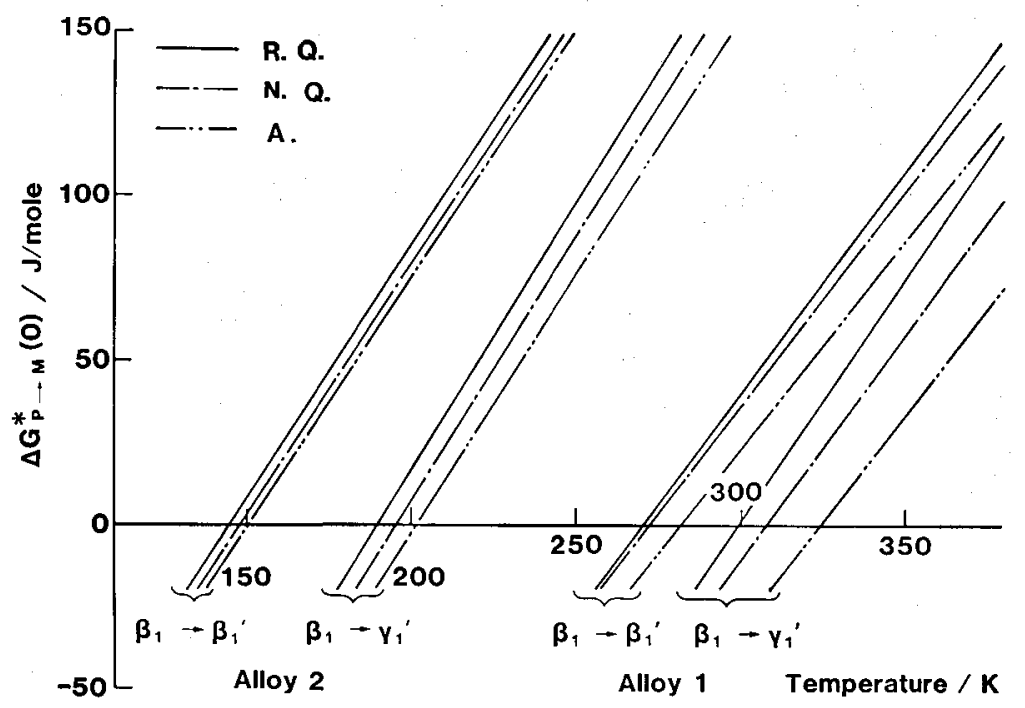

Fig. 14.

Effect of heat treatments on chemical free energy change $\Delta G^{*}$ vs. temperature $T$ for the $\beta_{1} \rightarrow \gamma_{1}^{\prime}$ and $\beta_{1} \rightarrow \beta_{1}^{\prime}$ transformations. 
different influences on the stability of each phase.

\subsection{Conclusive Elucidation}

Fig. 15 shows a schematic $\Delta G_{P \rightarrow M}$ vs. $T$ diagram. There are shown $\Delta G_{P \rightarrow M}$ vs. $T$ curves for two typical heat treatments for simplicity; R.Q. (solid lines denoted by $Q$ ) and $A$. specimens (dash-dotted lines denoted by A). Further, $T_{o}$ and $M_{s}$ temperatures and so on are denoted in such a way that $\mathcal{T}_{0}\left(Q, \gamma_{1}^{\prime}\right)$ is for the $\gamma_{1}^{\prime}$ martensite in the R.Q. specimen.

As derived from the calculation in Sec. 4.2, $T_{o}$ $\left(\gamma_{1}^{\prime}\right)$ is higher than $T_{o}\left(\beta_{1}^{\prime}\right)$ regardless of the heat treatments, but the difference between $T_{o}\left(Q, \gamma_{1}^{\prime}\right)$ and $T_{0}$ $\left(Q, \beta_{1}^{\prime}\right)$ is the smallest among the three heat treatments. Besides, as revealed from the $f-T$ diagrams (Fig. 8) and also from an optical microscopy observation of the transformation processes, supercooling $\Delta T_{s}$ or the driving force $\Delta G_{n}$ necessary for the nucleation of martensites is larger for the $\gamma_{1}^{\prime}$ martensite than for the $\beta_{1}^{\prime}$ martensite. Further, since the temperature hysteresis for transf. 1 in alloy 2 has been found not to so markedly vary with heat treatment, it may be assumed that the variation of $\Delta T_{s}$ or $\Delta G_{n}$ with heat treatment for the $\gamma_{1}^{\prime}$ and $\beta_{1}^{\prime}$ martensites is quite small, i.e., $\Delta T_{s}(Q) \simeq \Delta T_{s}(A)$ or $\Delta G_{n}(Q) \simeq \Delta G_{n}(A)$.

The above circumstances may bring about the following results. In R.Q. specimen whose $T_{o}\left(Q, \gamma_{1}^{\prime}\right)$ is only slightly higher than $T_{o}\left(Q, \beta_{1}^{\prime}\right), M_{s}\left(Q, \gamma_{1}^{\prime}\right)$ may be lower than $M_{*}\left(Q, \beta_{1}^{\prime}\right)$. Thereby, the $\beta_{1}^{\prime}$ martensite was thermally formed in R.Q. specimens. On the other hand, in A. specimens whose $T_{o}\left(A, \gamma_{1}^{\prime}\right)$ is fairly higher than $T_{o}\left(A, \beta_{1}^{\prime}\right), M_{*}\left(A, \gamma_{1}^{\prime}\right)$ is higher than $M_{s}\left(A, \beta_{1}^{\prime}\right)$, and then the $\gamma_{1}^{\prime}$ martensite is ther-

Table 2. Variation, $\delta \Delta G_{P \rightarrow M}^{*}$, of the free energy change associated with transformation due to the change of heat treatments from R. Q. to A.

\begin{tabular}{ccc}
\hline Transformation & Alloy 1 at $340 \mathrm{~K}$ & Alloy 2 at $240 \mathrm{~K}$ \\
\hline$\beta_{1} \rightarrow \gamma_{1}^{\prime}$ & -39.8 & -21.8 \\
$\beta_{1} \rightarrow \beta_{1}^{\prime}$ & -20.8 & -11.0 \\
\hline
\end{tabular}

mally formed.

Furthermore, the thermally formed martensites in alloy 2 have been found to remain unchanged independent of the heat treatments. The reason is, of course, because $T_{o}\left(\gamma_{1}^{\prime}\right)$ is fairly higher than $T_{o}\left(\beta_{1}^{\prime}\right)$ and therefore $M_{s}\left(\gamma_{1}^{\prime}\right)$ is always higher than $M_{s}\left(\beta_{1}^{\prime}\right)$. This fact is originated from the large difference in $G$ between the two concerned martensites. Thus, it may be concluded that $M_{s}$ temperature is a decisive factor for determining the kind of thermally formed martensites; the martensite with the highest $M_{s}$ temperature is thermally formed. The fact that the $\beta_{1}$ parent in N.Q. specimens has transformed sometimes into the $\beta_{1}^{\prime}$, a mixture of the $\beta_{1}^{\prime}$ and $\gamma_{1}^{\prime}$, or the $\gamma_{1}^{\prime}$, is simply because $M_{s}$ temperatures for the two martensites are almost the same.

A similar phenomenon is expected to occur also in other alloy systems in which the kind of thermally formed martensite phases changes with alloy composition or heat treatment, and the reason for the phenomenon may similarly be explained. In fact, the present authors have recently obtained an experimental evidence for such an explanation on the appearance of two martensite phases in a $\mathrm{Au}-47.5 \mathrm{at} \%$ Cd alloy. ${ }^{41}$ Further, it has been reported in $\mathrm{Ti}-\mathrm{Ni}$ alloys that the R-phase transformation occurs prior to the ordinary martensitic transformation through a heat treatment which lowers the normal $M_{s}$ temperature. ${ }^{42)}$ Moreover, Muto et al. have recently found in $\mathrm{Fe}_{3} \mathrm{Pt}$ alloys that a BCT martensite phase is formed in a disordered condition, while a FCT martensite phase is formed in an ordered condition. ${ }^{43}$ ) These phenomena are believed to be explained in a similar way to the present one.

\section{Conclusion}

An investigation has been carried out on the effect of three kinds of heat treatments, (a) rapid quench, (b) normal quench, and (c) low temperature aging after (b), on thermal martensitic transformations in



Fig. 15.

Schematic diagram showing the effect of heat treatment on chemical free energy change $\Delta G_{P \rightarrow M}$ vs. temperature $T$ (see text for details). 
monocrystallines of $\mathrm{Cu}-14.1 \mathrm{Al}-4.2 \mathrm{Ni}$ (alloy 1) and Cu-14.7 Al-4.2Ni (alloy 2) alloys, which has been done by means of DSC measurements, optical microscopy and tensile tests of martensitic specimens. As a result, the followings have been found.

(1) Transformation temperatures in both the alloys were raised in the order of (a), (b) and (c) treatments. In alloy 2 , the $\gamma_{1}^{\prime}$ martensite was thermally formed regardless of the heat treatments. However, thermally formed martensites in alloy 1 were found to vary with the heat treatments; i.e., the $\beta_{1}^{\prime}$ or the mixture of $\beta_{1}^{\prime}$ and $\gamma_{1}^{\prime}$ in (a), the $\beta_{1}^{\prime}$, the mixture of $\beta_{1}^{\prime}$ and $\gamma_{1}^{\prime}$ or the $\gamma_{1}^{\prime}$ in (b) and the $\gamma_{1}^{\prime}$ in (c).

(2) The shifts of equilibrium temperatures for the $\beta_{1} \rightleftarrows \gamma_{1}^{\prime}$ and $\beta_{1} \rightleftarrows \beta_{1}^{\prime}$ transformations were estimated by utilizing the data of stress-induced martensitic transformations and associated pseudoelasticity.

By taking into consideration the shifts of these equilibrium temperatures and supercooling necessary for the nucleation of the $\beta_{1}^{\prime}$ and $\gamma_{1}^{\prime}$ martensites as well, the reason for the change of martensite phases with heat treatment was well explained in terms of a simple scheme that the martensite phase with the highest $M_{s}$ temperature changes from the $\beta_{1}^{\prime}$ to the $\gamma_{1}^{\prime}$ with changing heat treatment from (a) to (c) via (b).

\section{Acknowledgements}

The authors are grateful to Mr. Y. Nakai, now at Sumitomo Electric Ind., Ltd. for assistance in tensile testing and optical microscopy of tensile specimens, Mr. A. Tanaka, now at Showa Electric Wire \& Cable Go., Ltd., for DSG measurements and optical microscopy of the DSC specimens, and Mr. Y. Tsuzuki, now at The Furukawa Electric Co., Ltd., for drawing the $f-\mathcal{T}$ diagram. The present investigation was partly supported by the Research Project of ISIR, Osaka University, on "Development of New Materials for Energy Conversion".

\section{REFERENCES}

1) G. Occampo and B. Dubois: Mém. Etud. Sci. Rev. Métall., 24 (1979), 53.

2) P. R. Swann and H. Warlimont: Acta metall., 11 (1963), 1099 .

3) J. Jellison and E. P. Klier: Trans. AIME, 233 (1965), 1694

4) R. S. Toth and H. Sato: Acta metall., 16 (1968), 413.

5) H. M. Ledbetter and C. M. Wayman: Metall. Trans., 3 (1972), 2349.

6) T. Tadaki and K. Shimizu: Trans. Jpn. Inst. Met., 18 (1977), 735.

7) T. Tadaki, Y. Katano and K. Shimizu: Acta metall., 26 (1978), 883.

8) S. Vatanayon and R. F. Heheman: Shape Memory Effects in Alloys, ed. by J. Perkins, Plenum Press, New York, (1975), 115.

9) H. Shumann: Arch. Eisenhüttenwes., 38 (1967), 647.

10) H. Shumann: Arch. Eisenhüttemwes., 40 (1969), 1027.

11) M. S. Wechsler and T. A. Read: J. Appl. Phys., 27 (1956), 194.

12) B. S. Subramanya, G. S. Baker, D. S. Lieberman and T. A.
Read: J. Aust. Inst. Met., 6 (1961), 3.

13) N. Nakanishi and G. M. Wayman: Trans. Jpn. Inst. Met., 4 (1963), 179.

14) T. Suzuki, M. Takagi, A. Nagasawa and N. Nakanishi: J. Mater, Sci., 6 (1981), 3013.

15) D. Hull and R. D. Garwood: J. Inst. Met., 86 (19571958), 485

16) S. Sugino, N. Nakanishi and H. Mitani: Trans, Jpn. Inst. Met., 29 (1965), 751.

17) H. Sakamoto, Y. Nakai and K. Shimizu: Trans. Jpn. Inst. Met., 28 (1987), 264.

18) K. Otsuka and K. Shimizu: Phil. Mag., 24 (1971), 481.

19) K. Otsuka, T. Nakamura and K. Shimizu: Trans. Jpn. Inst. Met., 15 (1974), 211.

20) K. Otsuka and K. Shimizu: Trans. Jpn. Inst. Met., 15 (1974), 103.

21) K. Otsuka and K. Shimizu: Jpn. J. Appl. Phys., 8 (1969), 1196.

22) G. V. Kurdjumov: J. Iron Steel Inst., (1960), May, 26.

23) G. V. Kurdjumov and L. G. Khandros: Doklady $A \mathcal{N}$, 66 (1949), 211.

24) K. Otsuka, M. Takahashi and K. Shimizu: Metall. Trans., 4 (1973), 2003

25) K. Otsuka, G. M. Wayman, K. Nakai, H. Sakamoto and K. Shimizu: Acta metall., 24 (1975), 207.

26) H. Sakamoto, K. Shimizu and K. Otsuka: Proc. JIMIS-1 (ICOMAT-76), Suppl. to Trans. Jpn. Inst. Met., 17 (1976), 245.

27) G. Génin, J. L. Macqueron, M. Mantel, G. Auguet, E. Cesai, Ll. Manôsa, A. Planes, J. Ortin, C. Picornell, C. Segui and V. Torra: Proc. Int. Conf. on Martens. Transf., (ICOMAT-86, Nara), Japan Inst. Met., Sendai, (1987), 794

28) J. Van Humbeeck, D. Van Hull, L. Delaey, J. Ortin, C. Segui and V. Torra: Trans. Jpn. Inst. Met., 28 (1987), 383.

29) J. W. Christian: The Theory of Transformations in Metals and Alloys, Pergamon Press, Oxford, (1965), Chap. 23.

30) J. B. Lightstone: Acta metall., 15 (1967), 25.

31) L. Delaey, J. Ortin and J. V. Humbeeck: Proc. Int. Conf. on Phase Transf., Inst. Metals, Cambridge, (1987), 60.

32) G. V. Kurdjumov: J. Metals, 11 (1959), 449.

33) D. P. Dunne and C. M. Wayman: Metall. Trans., 4 (1973), 137.

34) D. P. Dunne and C. M. Wayman: Metall. Trans., 4 (1973), 147.

35) Y. Nakata, T. Tadaki and K. Shimizu: Trans. Jpn. Inst. Met., 26 (1985), 646.

36) H. C. Tong and C. M. Wayman: Acta metall., 22 (1974), 887.

37) H. Sakamoto and K. Shimizu: Proc. Int. Symp. on NonEquilibrium Solid Phases of Metals and Alloys '88, Suppl. to Trans. Jpn. Int. Met., 29 (1988), 241.

38) H. Sakamoto and K. Shimizu: Trans. Jpn. Inst. Met., 28 (1987), 715.

39) P. Wollants, M. De Bonte and J. R. Roos: Z. Metallkd., 70 (1979), 113

40) K. Otsuka and K. Shimizu: Proc. Int. Conf. on SolidSolid Phase Transformations, Metall. Soc. AIME, New York, (1981), 1267.

41) H. Sakamoto, Y. Tsuzuki and K. Shimizu: Proc. MRS Int. Conf. on Advanced Materials, Mater. Res. Soc., Pittsburgh, (1989), in press.

42) S. Miyazaki and K. Otsuka: Metall. Trans. A., 17A (1986), 53.

43) S. Muto, R. Oshima and F. E. Fujita: Metall. Trans. A, 19A (1988), 2723. 